\title{
Optimal capacitor placement in distribution networks using Genetic Algorithm
}

\author{
Majid Davoodi ${ }^{1}$, Mohsen Davoudi ${ }^{2}$, Iraj Ganjkhany ${ }^{2}$ and Ali Aref $^{3}$ \\ ${ }^{1}$ Department of Electrical Engineering, Takestan Branch, Islamic Azad University, Takestan, Iran. \\ ${ }^{2}$ Department of Electrical Engineering, Abhar Branch, Islamic Azad University, Abhar, Iran. \\ ${ }^{3}$ Department of Electrical Engineering, Takestan Branch, Islamic Azad University, Takestan, Iran. \\ majid.davoodi@yahoo.com,mohsen.davoudi@polimi.it, iraj_ganjkhany@yahoo.com, ali.aref89@yahoo.com
}

\begin{abstract}
One of the on-going studies in the power distribution system is optimal placement of capacitors to which designers pay attention since many years ago. The most important benefit of capacitor placement is loss reduction, voltage profile improvement, increment of power factor and freeing up the power system capacity. This paper presents a new approach for capacitor allocation in a sample distribution system. The problem formulation considers most of effective parameters in the capacitor installation. One of the distinguished characteristics of the implementation of the Genetic Algorithm for optimal capacitor placement in distribution system is its multifunction capability. The proposed method on capacitor placement and detecting optimum capacitance has been implemented and tested in a 9-bus IEEE sample network in DIGSILENT and MATLAB environments.
\end{abstract}

Keywords: Capacitor placement, Genetic algorithm, Optimization.

\section{Introduction}

The majority of the energy losses happen in the distribution and transmission systems. The losses in the power system are produced by reactive current in the transmission lines. The reactive power is manufactured in the power generation sections and is engrossed transmission line by transferring. This problem can increase current in lines. This problem can be rise up with production of power in vicinity of the consumers. Power capacitors are the main equipment that can produce reactive power needed by consumers. Now, we do not need to transfer reactive power and which leads to term of distribution system improvement.

There are many ways for capacitor placement and determination size of capacitors in power systems. The current methods are useful and practical but the majority of them only consider some of the effective parameters for this problem. The most important effective parameters in capacitor placement are:

- The cost of loss reduction

- The cost of voltage profile improvement

- The cost of freeing up system capacity

- The cost of capacitor capacity

- The cost of capacitor installation and maintenance

In earlier studies only some parts of the effective parameters in capacitor placement have been considered. For example Baran \& Wu (1989) studied only loss reduction and voltage profile enhancement. An attempt by Gallego et al. (2001) to optimize capacitor placement taken into account only loss reduction, voltage profile enhancement, freeing up the capacity of the generators and cost of capacitors. In the work of Das, (2008) only losses reduction, voltage profile enhancement and installation cost of capacitors were considered. Mahmoodianfard et al. (2011) investigated loss reduction and voltage profile enhancement. Ghose et al. (1998), has done capacitor placement only by considering losses reduction and the installation cost of the capacitors.

This study takes more influencing factors into account in capacitor placement compare to the previous studies. This proposed method for capacitor placement leads to obtain all advantages of the capacitor placement in the power network. Furthermore, a couple of the optimization methods, which have been used in the other works for optimal capacity placement, are presented shortly in the discussion including the comparison with the proposed method.

\section{Problem statement}

The capacitor placement problem is solved optimally considering many factors such as reduction of ohmic losses, voltage profile enhancement, freeing up the generators capacity, freeing up the distribution capacity, freeing up the distribution utilities starting from gathering voltage and current of all nodes in network by load distribution in DIGSILENT environment. Some candidate buses will be selected then by running genetic algorithm in MATLAB to detect the optimum place and capacitance of capacitors. The network then is tested by load distribution to check whether the problem requests are met and the indexes are converged. Steps for finding these optimum points are shown in the Fig. 1.

Genetic algorithm

Genetic algorithm (GA) is one of the optimization algorithms in the problem solving that inspired from natural reproduction in fresh life (Gary Boone \& HsiaoDong, 1993). In this paper the genetic algorithm is used for optimization with multi-objective target function. There are many ways for capacitor placement Such as Numerical programming which is one of the traditional methods to solve capacitor placement in the power distribution system. The current methods are complex and slow in computational point of view. Artificial methods 
Fig. 1. Flowchart of finding the optimum points

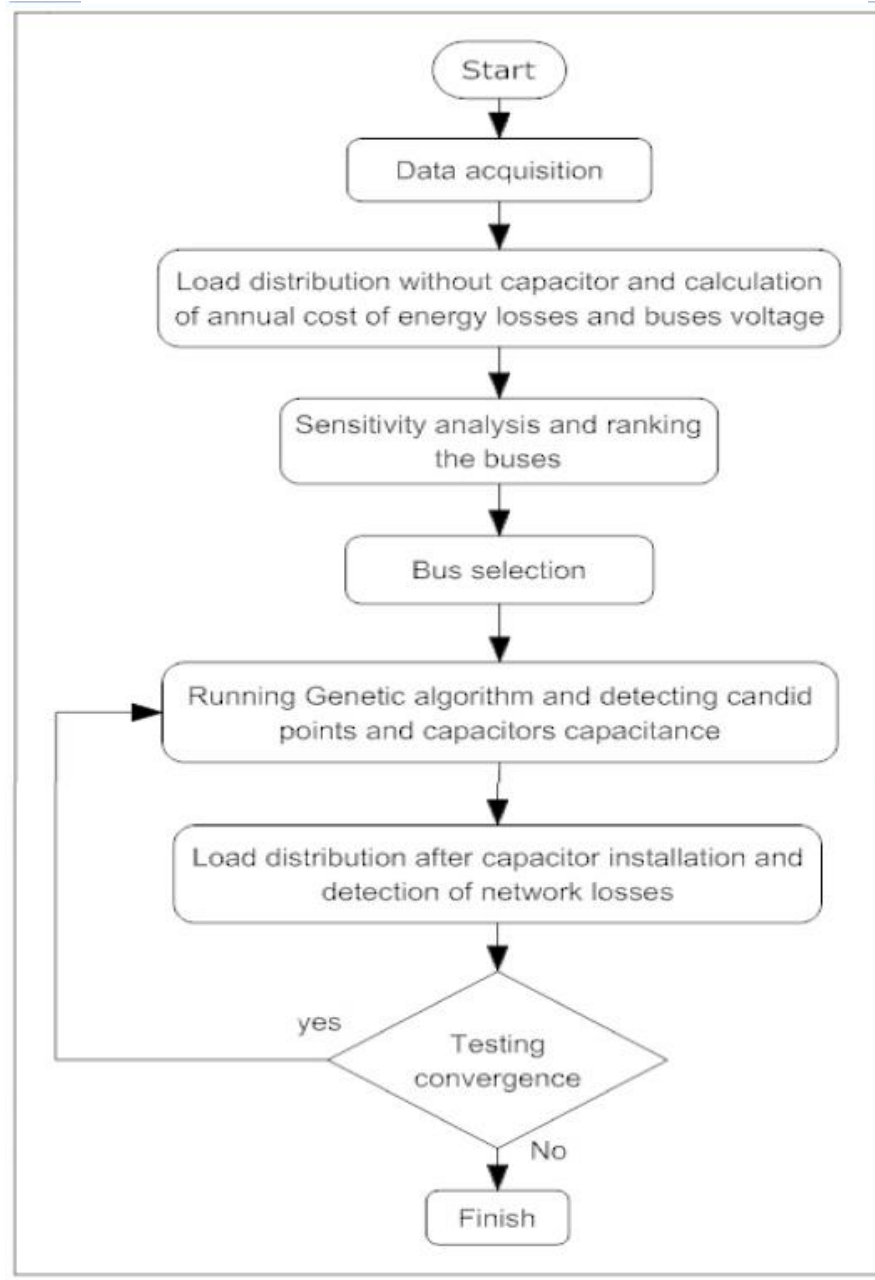

including TABU search, Steel plating, Particle crow theory, Fuzzy network theory, Body immune algorithm, Artificial networks and Genetic algorithm can be used for capacitor placement problem (Salama et al., 2000).

This study implements genetic algorithm for detection and placement of capacitors considering most of the influencing factors in the multi-objective target function. The advantage of the proposed method compare to the current intelligent methods such as Fuzzy method used by Prasad et al. (2007) and PSO (particle swarm optimization) used in (Xin-mei Yu et al., 2004) is that it converges faster.

Genetic algorithm optimization uses effectively the result of capacitor placement study on fresh life style and evolution process. Optimization process in GA include several steps: in the first step includes selecting the first population of the solution considering problem qualification and data amplitude. The first population includes small components called gene or chromosome. The second step includes the production of new population in each repetition using genetic operators. Production of new population continues until achieving optimum solution for the capacitor placement problem considering problem limitation. Fitness function in genetic algorithm is given by (1):

$$
\left\{E\left[m_{i}(s, t+1)\right] \geq \frac{u(s, t)}{\bar{f}(t)} \cdot m_{i}(s, t)\left(1-p_{c} \frac{d_{s}}{l-1}\right) \cdot\left(1-p_{n}\right)^{0(s)}\right\}(1)
$$

Where:

$m(s, t)$ : number of instances of schema $s$ in population at time $t$

$\bar{f}(t)$ : average fitness of population at time $t$

$u(s, t)$ : average fitness of instances of schema $s$ at time $t$

$p_{c}$ :probability of single point crossover operator

$P_{m}$ :probability of mutation operator

$l$ : length of individual bit strings

$0(s)$ : number of defined (non "*") bits in $s$

$d_{s}$ : distance between rightmost, leftmost defined bits in $s$

Genetic operators:

Perform evolution and optimization on genes in each generation by genetic operators. This operator can lead to production of new generation from the old one. The new generation produced by these operators is better than the old ones. Genetic operators include:

1. Selection: generation of the first population in confinement problem by selecting genes quite randomly, then selection of the genes that are effective in producing new generation.

2. Crossover: this operator can lead to change in gene bits. For example interchange of specific bits in one gene with similar bits in other gene.

\begin{tabular}{l} 
Children \\
\begin{tabular}{|r|c|c|c|c|c|c|c|c|c|c|}
\hline 1 & 1 & 1 & 0 & 1 & 0 & 0 & 1 & 0 & 0 & 0 \\
\hline 0 & 0 & 0 & 0 & 1 & 0 & 1 & 0 & 1 & 0 & 1 \\
\hline \hline
\end{tabular} \\
\hline \begin{tabular}{|c|c|c|c|c|c|c|c|c|c|c|}
\hline \hline 1 & 1 & 1 & 0 & 1 & 0 & 1 & 0 & 1 & 0 & 1 \\
\hline 0 & 0 & 0 & 0 & 1 & 0 & 0 & 1 & 0 & 0 & 0 \\
\hline
\end{tabular}
\end{tabular}

3. Mutation: this operator changes one or more bits in one gene randomly to improve its condition, for example in one bit change 1 to 0 or 0 to 1 .

Children

\begin{tabular}{|c|c|c|c|c|c|c|c|c|c|c|c|}
\hline 1 & 1 & 1 & 0 & & $0 \mid 0$ & $C$ & & & & 0 & 0 \\
\hline \multicolumn{12}{|c|}{ Parents } \\
\hline 1 & 1 & 1 & 0 & 1 & ( & 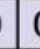 & & & & & 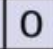 \\
\hline
\end{tabular}

The Proposed Method

In this study to compute the capacitance and location of capacitor in the distribution system, the genetic algorithm has been used. The target function of the genetic algorithm is a cost function that includes most of the known parameters such as cost of decreasing losses, cost of voltage profile enhancement, cost of capacitor installation that include fix and variable cost that is based on capacitor capacitance and has following equation: $F_{\operatorname{cost}(x)}=K_{1} C_{l}+K_{2} C_{v}+K_{3} C_{i}+K_{4} C_{f}+K_{5} C_{r}\left(\frac{\$}{K w h}\right)(2)$ 
Table1. The 9-bus IEEE network line data set

\begin{tabular}{|c|c|c|c|}
\hline Sen.bus & Res.bus & $\mathrm{X}(\mathrm{ohm})$ & $\mathrm{R}$ (ohm) \\
\hline 0 & 1 & 0.4127 & 0.1233 \\
\hline 1 & 2 & 0.605 & 0.014 \\
\hline 2 & 3 & 1.205 & 0.7463 \\
\hline 3 & 4 & 0.6084 & 0.6984 \\
\hline 4 & 5 & 1.7276 & 1.9831 \\
\hline 5 & 6 & 0.7886 & 0.9053 \\
\hline 6 & 7 & 1.164 & 2.0552 \\
\hline 7 & 8 & 2.716 & 4.7953 \\
\hline 8 & 9 & 3.0264 & 5.3434 \\
\hline
\end{tabular}

Table 2. The 9-bus IEEE network buses data set

\begin{tabular}{|l|l|l|}
\hline No.bus & $\mathrm{P}(\mathrm{Kw})$ & $\mathrm{Q}(\mathrm{Kvar})$ \\
\hline 1 & 1840 & 460 \\
\hline 2 & 980 & 340 \\
\hline 3 & 1790 & 446 \\
\hline 4 & 1598 & 1840 \\
\hline 5 & 1610 & 600 \\
\hline 6 & 780 & 110 \\
\hline 7 & 1150 & 60 \\
\hline 8 & 980 & 130 \\
\hline 9 & 1640 & 200 \\
\hline
\end{tabular}

where:

$\mathrm{F}_{\operatorname{cost}(\mathrm{x})}:$ target function

$C_{l}$ : cost of losses in the network

$C_{v}$ : cost of voltage profile enhancement

$C_{i}$ : cost of each capacitor installment

$C_{f}$ : cost of capacitor (based on it's capacitance)

: cost of generators capacity, lines and distribution utilities development,

$K_{1}$ : coefficient of transferring losses to cost

$K_{2}$ : coefficient of transferring voltage profile enhancement to cost

$K_{3}$ : coefficient of transferring each capacitor installation to cost

$K_{4}$ :coefficient of transferring capacitor capacitance to cost $K_{5}$ : coefficient of transferring capacity of system an apparatus development to cost

As an example for calculating the cost of losses in the network, the differences of losses in each bus before and after capacitor installation is computed in DIGSILENT. This difference is used as $K_{1}$ coefficient multiplied in the voltage profile enhancement cost. The other influencing cost parameters are calculated as like for this one. The $K_{i}$ are the coefficients of the target function given to DIGSILENT as a matrix called coefficients matrix, which

Fig. 2. The 9-bus IEEE single line diagram

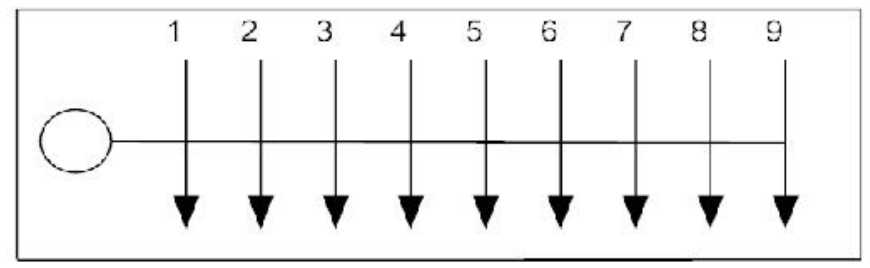

is discussed in the next sections. The Limitations faced while running the algorithm are as follows:

1. Limitation of the buses voltage: $V_{\min }<V<V_{\max }$, $V_{\min }<V<V_{\max }$ the buses voltage after capacitor installations have not to be more than detected boundaries.

2. Limitation of the capacitor bank: used capacitor bank has a fixed capacitance and can't be varied.

In this study a sample network was selected to implement the optimal capacitor placement and capacitance detection by the proposed algorithm considering the most of the known influencing factors (Majid davoodi et al., 2012).

Table 3. Destination function coefficients

\begin{tabular}{|c|l|c|}
\hline Coefficient & \multicolumn{1}{|c|}{ Parameter } & $\begin{array}{c}\text { Coefficient applied to } \\
\text { each parameter in the } \\
\text { destination function }\end{array}$ \\
\hline$K_{1}$ & Loss reduction & $25 \%$ \\
\hline$K_{2}$ & Voltage profile & $15 \%$ \\
\hline$K_{3}$ & $\begin{array}{l}\text { Capacitor } \\
\text { installation cost }\end{array}$ & $25 \%$ \\
\hline$K_{4}$ & $\begin{array}{l}\text { Capacitor } \\
\text { capacitance cost }\end{array}$ & $20 \%$ \\
\hline$K_{5}$ & $\begin{array}{l}\text { System over } \\
\text { capacitance cost }\end{array}$ & $15 \%$ \\
\hline
\end{tabular}

Fig. 3. A general form of genes bits used in the genetic algorithm

\begin{tabular}{|l|l|l|l|l|l|}
\hline 1 & 2 & 3 & 4 & $\ldots \ldots \ldots \ldots \ldots \ldots . .$. & $\mathrm{n}$ \\
\hline
\end{tabular}

The simulated distribution network

In this study to compare the results achieved from the capacitor placement in the distribution system a standard 9-bus IEEE power network is selected (its single line diagram is shown in Fig. 2.This network has one voltage source and a data set about its lines and buses shown in Tables 1 and 2 .

The genes (chromozóm) used in the genetic algorithm for solving the capacitor placement problem in this study has $n$ member. Each member represents one bus in the network. Values of these buses in the th position show the capacitance of the installable capacitors in the $l^{\text {th }}$ bus. A general form of genes is shown in Fig.3. The first generation of the genes is initialized randomly in the start of the genetic algorithm.

\section{Simulation results}

In this study, the optimal placement and capacitance detection of the capacitors using the genetic algorithm including the most of the influencing parameters in its target function has been implemented in a 9-bus IEEE network.

Input matrix of target function including coefficients such as cost of losses reduction, voltage profile increment, transferring capacitor installment to cost, transferring capacitor capacitance to cost and transferring over capacitance of systems and apparatus to cost that called $K_{1}, K_{2}, K_{3}, K_{4}, K_{5}$ respectively in the target function. The parameters consider environmental, economical
Research article

CCIndian Society for Education and Environment (iSee)
"Power system capacitor" http://www.indjst.org
M.Davoodi et al. 
conditions such as weather and power market as well (see Table 3).

Table 4. Result of running algorithm on a 9-bus IEEE network

\begin{tabular}{|c|c|c|c|}
\hline Bus & Capacitor size & $\begin{array}{c}\text { Number of } \\
\text { capacitor }\end{array}$ & PR \\
\hline 1 & KVar300 & 1 & \multirow{2}{*}{0.88438} \\
\hline 2 & KVar600 & 2 & \\
\hline 5 & KVar300 & 1 & \\
\hline 6 & KVar1200 & 4 & \\
\hline
\end{tabular}

These coefficients are computed based on their importance and influence on decreasing costs given as a column matrix to DIGSILENT. Notice that these factors may be changed in each network (Aref et al., 2012a,b). Based on these factors, target function is created and the genetic algorithm based optimization method is implemented. The proposed method has been developed in DIGSILEN and MATLAB environments. In MATLAB by assigning values to the algorithm, the results of the standard 9-bus IEEE network are shown in Table 4.

In Table 4, PR is the index of losses decrement which in the genetic algorithm is the ratio of total losses in the system after capacitor placement to all of losses before capacitor placement:

$P R=\frac{P_{\text {lossnew }}}{P_{\text {loss }}}(3)$

Where $P_{\text {lossnew }}$ and $P_{\text {loss }}$ refer to losses after capacitor placement and before it respectively.

Achieved result from losses indexes are as follows:

- PR <1: capacitor placement results in costs reduction.

- $P R=1$ : capacitor placement does not have any effect on the network costs.

- PR > 1: capacitor placement results in costs increment. When there is no capacitor placement in the network, the value of the cost function is $F_{\operatorname{cost}(x)}=330125$ and after capacitor placement based on Table $4, \mathrm{~F}_{\operatorname{cost}(\mathrm{x})}=308900$. In the Table 5 detecting the location and capacitance of the capacitors based on the influencing factors using genetic algorithm is shown with comparison to the other methods. It can be seen that the results of the proposed method has lower cost than others.

Table 5. Comparison proposed capacitor placement with other methods

\begin{tabular}{|c|c|c|c|c|c|}
\hline Bus & $\begin{array}{c}\text { Solution } \\
1\end{array}$ & $\begin{array}{c}\text { Solution } \\
2\end{array}$ & $\begin{array}{c}\text { Solution } \\
3\end{array}$ & $\begin{array}{c}\text { Solution } \\
4\end{array}$ & $\begin{array}{c}\text { Genetic } \\
\text { algorithm }\end{array}$ \\
\hline 1 & 300 & 300 & 300 & & 300 \\
\hline 2 & 300 & & & 300 & 600 \\
\hline 3 & & 300 & & & \\
\hline 4 & & & & 600 & \\
\hline 5 & 300 & 300 & 900 & & 300 \\
\hline 6 & 1200 & 1200 & 1200 & 1200 & 1200 \\
\hline 7 & & & & & \\
\hline 8 & & & & & \\
\hline$\frac{9}{\sigma}$ & & & & & \\
\hline$\frac{5}{F_{\operatorname{cost}(x)}}$ & 308964 & 309035 & 309041 & 309049 & 308900 \\
\hline
\end{tabular}

\section{Discussion}

Optimal placement of capacitor in distribution systems are among the on-going problems that many studies have been performed in this area. Due to importance of this problem, different methods are used to find the optimal solution. In this section a couple of optimization methods (PSO Algorithm and Plant Growth Simulation Algorithm) used in the previous works are shortly explained:

1. The particle swarm optimizer (PSO) algorithm firstly was presented by Dr. Kennedy and Dr. Eberhart. It is a random evolution method based on intelligent search of the group birds. It has quick convergence speed and optimal searching ability for solving large-scale optimization problems. It emulates the 'flocking' or 'swarming' behavior seen in animals such as birds and insects. As such, the 'swarm' is initialized with a population of random solutions, and successive generations of particles are then 'flown' through this solution space at a certain velocity. Prakash \& Sydulu (2007) used PSO algorithm for optimal capacitor placement in distribution system.

2.Plant Growth Simulation Algorithm (PGSA) is another method that is used for optimal capacitor allocation. This algorithm is based on the plant growth process, in the way that a plant grows a trunk from its root, then branches will grow from the nodes on this trunk, and then new branches will grow from old branches and this process continue until optimal response be found. Srinivasas et al. (2011) used PGSA for optimal capacitor placement in distribution system, but target function in the previous works checks only two parameters (comprehensive voltage profile and loss reduction) while leaving the other factors.

\section{Conclusion}

In this paper the optimal capacitor placement and capacitance computation in the power distribution networks using a proposed method based on genetic algorithm considering the majority of the influencing factors in its multi-objective target function have been studied. In the first step, the coefficient of the target function is got weighted in a column matrix in which the value of each factor possibly may be different for each network. Entering the factors of the target function in DIGSILENT environment and running genetic algorithm, the results have been achieved. This study shows that in optimal placement of capacitors and detecting optimum capacitance considering most of the important factors (such as loss reduction, voltage profile enhancement, cost of installation and capacitor cost based on it's capacitance), using genetic algorithm the best values to target function can be achieved.

\section{References}

1. Ali Aref, Mohsen Davoudi and Majiddavoodi (2012a) Optimal placement and estimation of dg capacity in distribution network's using genetic algorithm-based method. Indian J. Sci. Technol. 5(3), 2281-2286.
Research article

CIndian Society for Education and Environment (iSee)
"Power system capacitor"

http://www.indjst.org
M.Davoodi et al.
Indian J.Sci.Technol. 
2. Ali Aref, Mohsen Davoudi, Farzad Razavi and Majid Davoodi (2012b) Optimal DG placement in distribution network's using intelligent systems. Energy \& Power Eng. (EPE). 4(2), 92 - 98.

3. Baran ME and Wu FF (1989) Network reconfiguration in distribution systems for loss reduction and load balancing. IEEE Trans. Power Delivery. 4(2), pp: 1401.

4. Das D (2008) Optimal placement of capacitor in radial distribution system using a Fuzzy-GA method. Indian Elect. Power \& Energy Sys. 30, 361-367.

5. Gallego RA, Monticelli Aj and Romero R(2001) Optimal capacitor placement in radial distribution networks. IEEE Trans. Power Sys. 16(4), 630-637.

6. Gary Boone \& Hsiao-Dong Chiang (1993) Optimal capacitor placement in distribution systems by genetic algorithm. Elect. Power \& Energy. 15(3), 155 - 161.

7. Ghose T, Goswami SK and Basu SK (1998) Energy loss reduction in distribution system by capacitor placement through combined GA-SA technique. IEEE Trans. Power Sys. 2, 502 - 505.

8. Mahmoodianfard $F$, Abyaneh $H A$, Salehi $H$ and Vahabzadeh A (2011) Optimal capacitor placement for loss reduction. IEEE Trans. Modern Elect. Power Sys. pp:1-5.

9. Majid Davoodi, Mohsen davoudi, Ali aref and Irajganjkhany (2012) Body immune algorithm based method for optimal capacitor placement in power distribution networks. J. Basic \& Appl. Scientific Res., April.

10.PrakashK and Sydulu M (2007) Particle swarm optimization based capacitor placement on radial distribution systems. IEEE Power Engg. Soc. 1 - 5.

11.PrasadPV, Sivanagaraju $S$ and Sreenirasulu N (2007) A Fuzzy - Genetic algorithm for optimal capacitor placement in radial distribution systems. ARPN J.Eng. \& Appl. Sci. 2(3), 28-32.

12.Salama MMA, $\mathrm{Ng} \mathrm{HN}$ and Chikhani AY (2000) Classificaton of capacitor allocation techniques. IEEE Trans. Power Delivery.15(1),387 - 392.

13.Srinivasas Rao $R$, Narasimham SVL and Ramalingaraju M (2011) Optimal capacitor placement in a radial distribution system using plant growth simulation algorithm. Elsevier, Elect.I Power \& Energy Sys. 33(5), 1133 - 1139.

14.Xin-mei Yu, Xin-Yin Xiong and Yao-wu Wu (2004) A PSO-based approach to optimal capacitor placement with harmonic distortion consideration. Elect. Power Sys. Res.71(1), 27-33. 